\title{
Relación entre el estado nutricional y el síndrome metabólico en adultos
}

\author{
María J osé Romero Ausfeld', Ana Aguilar Rabito" \\ I. Unidad de Obesidad del Hospital de Clínicas, Universidad Nacional de Asunción. Nutricionista colaboradora. \\ II. Facultad de Ciencias Químicas, Universidad Nacional de Asunción.
}

Cómo referenciar este artículo/ How to reference this article:
Romero MJ, Aguilar A. Relación entre el estado nutricional y el síndrome metabólico en adultos. Mem. Inst. Investig. Cienc. Salud. $2015 ; 13(2): 67-77$

\section{R E S U M E N}

El síndrome metabólico constituye una agrupación de factores como son la obesidad abdominal, dislipidemia, glucemia y presión arterial elevadas, implicados en el desarrollo de la diabetes mellitus y enfermedades cardiovasculares. El estado nutricional refleja la condición física en la que se encuentra un individuo, evaluado comúnmente por el Índice de Masa Corporal (IMC) o Índice de Quelet. El objetivo de este estudio fue evaluar la relación entre el estado nutricional y el síndrome metabólico en adultos de ambos sexos. Estudio transversal analítico con datos secundarios de sujetos que frecuentaron el parque de la salud del Instituto de Previsión Social (Asunción, Paraguay) en el mes de setiembre del 2.011, realizado en 98 sujetos con rango de edad de 34 a 51 años. La prevalencia del síndrome metabólico fue del 25,5\%. En mujeres con síndrome metabólico fueron más frecuentes los criterios de circunferencia de cintura $(p<0,001)$ y triglicéridos $(p<0,001)$ aumentados, en hombres el colesterol-HDL bajo $(p=0,001)$, la glucemia $(p=0,007)$ y presión arterial elevadas $(p=0,002)$. El estado nutricional según índice de masa corporal indicó un mayor porcentaje de exceso de peso en hombres $(89,6 \%)$. Al relacionar el estado nutricional con el síndrome metabólico, en general, la mayoría de los sujetos obesos presentaron síndrome metabólico $(p<0,001)$; por sexo se observó que la mayoría de las mujeres tenían sobrepeso y que los hombres con obesidad presentaron un mayor porcentaje de síndrome metabólico $(p=0,001)$. El estado nutricional y el síndrome metabólico se incrementan de manera proporcional, observándose una mayor prevalencia de síndrome metabólico en mujeres con sobrepeso y en hombres con obesidad. La prevalencia del síndrome metabólico fue del 25,5\% siendo menor el síndrome metabólico premórbido $(22,4 \%)$.

Palabras clave: síndrome metabólico, estado nutricional, enfermedades cardiovasculares.

\section{Relation between nutritional status and metabolic syndrome in adults}

\section{A B S T R A C T}

Metabolic syndrome is the result of various factors such as abdominal obesity, dyslipidemia, high blood glucose levels and high blood pressure, which can cause diabetes mellitus and cardiovascular diseases. The objective of this study was to evaluate the relationship between nutritional status and metabolic syndrome in adults of both sexes. Cross-sectional study with secondary data from subjects who frequented the "Instituto de Prevision Social" health park in September of 2011; 98 subjects from 34 to 51 years old were evaluated. The prevalence of the metabolic syndrome was $25.5 \%$. In women with metabolic syndrome the circumference waist criteria were more frequent $(p<0,001)$ and increased triglyceride $(p<0,001)$, in men low cholesterol-HDL 
$(p=0,001)$, glycemia $(p=0,007)$ and high blood pressure $(p=0,002)$. The nutritional status according to the body mass index indicated a higher percentage of overweight in men $(89.6 \%)$. Relating the nutritional status with the metabolic syndrome in general most of the obese subjects presented metabolic syndrome $(p<0,001)$ It it was observed by sex that most of the overweight women and obese men presented a higher percentage of metabolic syndrome $(p=0,001)$. The nutritional status and the metabolic syndrome increased in proportional way, observing a higher prevalence of metabolic syndrome in women with overweight and men with obesity. The prevalence of the metabolic syndrome was $25.5 \%$ being lower the metabolic premorbid syndrome $(22.4 \%)$.

Key words: metabolic syndrome, nutritional status, cardiovascular diseases

\section{NTRODUCCI ÓN}

La prevalencia de enfermedades crónicas ha aumentado dramáticamente en los últimos años hasta convertirse en una de las principales causas de discapacidad y muerte a nivel mundial $(1,2)$. Datos recientes revelan que, de forma paralela, con el aumento del sobrepeso y la obesidad también se ha observado un incremento en las prevalencias de diabetes mellitus 2 (DM2), hipertensión arterial (HTA), y colesterol elevado, con cifras cercanas a $10 \%, 40 \%$ y $39 \%$ respectivamente (3). Cada una de estas alteraciones de forma independiente suponen un riesgo para la salud del individuo a mediano y largo plazo, sin embargo, el riesgo se incrementa aún más cuando estas alteraciones se manifiestan en conjunto.

Es así que en 1.988, Reaven describió un conjunto de alteraciones relacionadas con las enfermedades cardiovasculares, en la cual la Resistencia a la Insulina (IR) actuaba como mecanismo fisiopatológico de base, esta patología se conoce hoy en día como Síndrome Metabólico (SM).

En el estudio original, la obesidad no fue considerada como un factor de riesgo para esta enfermedad. Sin embargo, diversos estudios epidemiológicos realizados posteriormente demuestran que además de la $I R$, la obesidad central presenta una fuerte asociación como factor potenciador de la misma $(4,5)$.

Hasta la fecha, el Adult Treatment Panel III (ATP III) del National Cholesterol Education Program (NCEP), la International Diabetes Federation (IDF) y World Health Organization (WHO), tres organizaciones conformadas por grupos de expertos, han establecido criterios para definir el SM entre los cuales han considerado a la obesidad central como indicador de IR y factores como la dislipidemia, HTA, vinculados con la enfermedad cardiovascular y la DM2 $(6,7)$. Los criterios más recientes fueron establecidos por la OMS en el 2.009 y modificados finalmente en el 2.010, que difieren de las demás recomendaciones debido a que excluyen a personas con DM2 y antecedente de enfermedad cardiovascular (ECV) considerando al SM como una condición premórbida (8).

Como toda patología crónica, la frecuencia de SM aumenta de forma paralela a la edad, observándose una mayor prevalencia después de los 40 años, siendo un factor de riesgo no modificable al igual que el origen étnico (afroamericanos), historia familiar de DM2 y mujeres en edad postmenopáusica. En cambio, el IMC mayor a $25 \mathrm{~kg} / \mathrm{m}^{2}$, el consumo excesivo de alcohol, hábito de fumar, estrés, alimentación rica en grasas y el sedentarismo, son otros factores que pueden ser corregidos adoptando estilos de vida saludables $(9,10)$.

Es bien sabido que la obesidad aumenta el riesgo de padecer complicaciones metabólicas, sin embargo, un reciente estudio realizado en España refiere que a pesar de que existen personas con obesidad, éstas pueden encontrarse metabólicamente sanas, estando ausentes las señales de inflamación crónica comunes en el sobrepeso, 
por lo que hay menor riesgo de padecer patologías relacionadas observándose un comportamiento similar del tejido adiposo al de personas con normopeso (11).

En el 2.011 se llevó a cabo en Paraguay la primera Encuesta Nacional de Factores de Riesgo y Enfermedades No Transmisibles (ENT). Los resultados preliminares de esta encuesta revelaron que $57,6 \%$ de la población sufría de sobrepeso y $22,8 \%$ de los paraguayos eran obesos. Alrededor del $10 \%$ eran diabéticos, $35 \%$ hipertensos y aproximadamente 2 de cada 10 presentaban alteraciones de los lípidos plasmáticos (12).

A pesar de los grandes esfuerzos realizados en el sector salud, aún no contamos con cifras nacionales que nos permitan tener una idea de la prevalencia de SM en la población paraguaya, sin embargo, estudios aislados realizados con muestras pequeñas y en otros grupos etarios, demuestran que los determinantes del SM están presentes desde edades tempranas. Riveros et al. hallaron que la frecuencia de SM en un grupo de 125 adolescentes de un colegio público fue menor al 1\%, sin embargo 32,8 \% presentó uno o más componentes del SM y el $88 \%$ de la muestra estudiada contó con antecedentes familiares de al menos un factor de riesgo para el desarrollo de SM, pudiendo con estos datos deducir una alta probabilidad de padecer SM en la edad adulta (13).

El informe de la situación mundial de las ENT de la OMS evidencia que los principales grupos de riesgo con mayor morbilidad son aquellos de países de ingresos bajos y medios, siendo más vulnerables a las enfermedades cardiovasculares como consecuencia de la detección tardía (1). A partir de ese marco conceptual, conocer la situación país adquiere importancia desde el punto de vista preventivo, tener una idea global de los principales grupos de riesgo nos permitirá diseñar e implementar estrategias de intervención temprana adaptadas a las características de los individuos a los cuales va dirigida.

Por lo mencionado anteriormente, el presente estudio pretendió identificar el síndrome metabólico utilizando la definición de la OMS 2.009 y relacionarlo con el estado nutricional de una población de sujetos que asiste al Parque de la Salud del Instituto de Previsión Social, dar a conocer la prevalencia del SM tanto por la definición de la OMS 2.009 como la de la OMS 2.010 o SMP (síndrome metabólico premórbido) en esta población, que a diferencia de los principales grupos de riesgo, tiene a favor que en su mayoría el nivel de ingresos es alto, identificar cuáles son sus riesgos para desarrollar a futuro enfermedad cardiovascular o DM2 e indagar cual sería la situación de salud/ enfermedad de nuestra población siendo que carecemos de estudios representativos que revelen la prevalencia del SM.

\section{MATERI ALES Y MÉTODOS}

Estudio transversal analítico con datos secundarios provenientes de un estudio denominado "Perfil lipídico como factor de riesgo cardiovascular y su relación con la actividad física en adultos que frecuentaron el Parque de la Salud del Instituto de Previsión Social", realizado durante los meses de setiembre y octubre del 2.011.

Se analizaron los datos de 98 sujetos de ambos sexos de 18 a 64 años en quienes se ha evaluado el estado nutricional mediante peso y talla y contasen con las mediciones necesarias para evaluar el síndrome metabólico.

Se consideró SM presente en aquellos sujetos que presentaban al menos tres de los cinco criterios citados a continuación: glucemia en ayunas $\geq 100 \mathrm{mg} / \mathrm{dl}$ o tratamiento antidiabético; presión arterial sistólica $\geq 130 \mathrm{mmHg}$ o diastólica $\geq 85 \mathrm{mmHg}$; colesterol $\mathrm{HDL}<40 \mathrm{mg} / \mathrm{dl}$ en los hombres o $<50 \mathrm{mg} / \mathrm{dl}$ en las mujeres; triglicéridos $\geq 150 \mathrm{mg} / \mathrm{dl}$; perímetro abdominal $\geq 102 \mathrm{~cm}$ para los hombres $0 \geq 88 \mathrm{~cm}$ para las mujeres. Los datos bioquímicos fueron determinados en un laboratorio privado mediante el método enzimático colorimétrico con equipo autoanalizador cinético-químico BT-Targa 3000 (Italiano). La presión arterial fue medida mediante un esfingomanómetro manual de 
marca San-Up (Argentina). El peso y la talla fueron medidos mediante una balanza de marca Balmak (Brasil) con capacidad máxima de $150 \mathrm{~kg}$ y $2 \mathrm{~m}$, con precisión de $100 \mathrm{~g}$ y $10 \mathrm{~mm}$, respectivamente.

Los datos fueron digitalizados en una planilla electrónica de Microsoft Office Excel $\circledast$ versión 2.007.

Los resultados se presentaron discriminados por sexo en frecuencias, promedios y desvío estándar o mediana, máximo y mínimo según el comportamiento de los datos, luego de la evaluación de la consistencia de los mismos.

Para comprobar si los valores eran significativamente diferentes unos de otros se utilizó la prueba estadística adecuada conforme cómo se encontraba la distribución de los datos, considerando un nivel de significancia estadística de $p \leq 0,05$.

Para evaluar la asociación entre las variables categóricas se utilizó la prueba $\mathrm{Chi}^{2}\left(\mathrm{X}^{2}\right)$, de Yates o Fischer según correspondiera. Y para evaluar las diferencias entre las variables numéricas se utilizó la prueba $t$ o la prueba de la mediana según el comportamiento de las variables, excepto para la edad que se decidió representar en mediana, máximo y mínimo por el tipo de variable.

Aspectos éticos de respeto y confidencialidad fueron considerados durante la investigación al ser utilizados los datos solo con fines de estadísticas generales, no vinculando nombres o identificación personal, y el estudio contó con la aprobación del Comité de Ética de la Facultad de Ciencias Químicas de la Universidad Nacional de Asunción.

\section{RESULTADOS}

La muestra estuvo constituida por 98 sujetos, de los cuales el $49 \% \quad(n=48)$ eran hombres y $51 \%(n=50)$ mujeres. La mediana de edad fue de 46 años (35-52 años) en los hombres y 44 años (31-52) en las mujeres. El $8,3 \%(n=4)$ de los hombres y $10,2 \%$ $(n=6)$ de las mujeres eran adultos mayores.

Se evaluó el estado nutricional según índice de masa corporal por sexo observándose un mayor porcentaje de exceso de peso en hombres $(n=43 ; 89,6 \%)$ que en mujeres $(n=32 ; 64 \%)(p=0,021)$.

Al analizar las características antropométricas se encontraron valores más altos de peso, talla y circunferencia de cintura en hombres $(p<0,001)$. En cuanto a las características clínicas de la muestra se observaron valores más altos de triglicéridos $(p=0,043)$ y presión arterial sistólica $(p=0,005)$ y de colesterol HDL disminuido $(p<0,001)$ en hombres (Tabla 1$)$.

Se observó diferencia entre la prevalencia de síndrome metabólico $(25,5 \% ; n=25)$ y síndrome metabólico premórbido $(22,4 \% ; n=22)$ en la muestra, siendo en su mayoría hombres (Tabla 2). 
Tabla 1. Características antropométricas y clínicas

\begin{tabular}{lllll}
\hline Variables & Total & Hombres & Mujeres & Valor $\mathbf{p}$ \\
\hline Peso $(\mathrm{kg})^{\mathrm{a}}$ & $80,9 \pm 16,8$ & $89,1 \pm 11,6$ & $73,2 \pm 17,5$ & $<0,001^{* *}$ \\
Talla $(\mathrm{m})^{\mathrm{a}}$ & $1,67 \pm 0,09$ & $1,74 \pm 0,062$ & $1,61 \pm 0,06$ & $<0,001^{* *}$ \\
IMC $\left(\mathrm{kg} / \mathrm{m}^{2}\right)^{\mathrm{a}}$ & $28,9 \pm 5,29$ & $29,6 \pm 3,8$ & $28,2 \pm 6,4$ & $0,205^{* *}$ \\
CC $(\mathrm{cm})^{\mathrm{a}}$ & $94,2 \pm 14,7$ & $101,4 \pm 10,4$ & $87,3 \pm 14,9$ & $<0,001^{* *}$ \\
C-HDL $(\mathrm{mg} / \mathrm{dl})^{\mathrm{a}}$ & $51,3 \pm 12,2$ & $46,7 \pm 10,7$ & $55,8 \pm 12,0$ & $<0,001^{* *}$ \\
Glicemia $(\mathrm{mg} / \mathrm{dl})^{\mathrm{b}}$ & $76(72,8-84,3)$ & $78,5(74-86)$ & $76(71-83)$ & $0,107^{*}$ \\
Triglicéridos $(\mathrm{mg} / \mathrm{dl})^{\mathrm{b}}$ & $132,5(89,3-214,5)$ & $160,5(105-235)$ & $108,5(72,8-$ & $0,043^{*}$ \\
& & & $182,8)$ & \\
PAS (mmHg) & $115(100-130)$ & $120(110-130)$ & $110(100-120)$ & $0,005^{\mathrm{b}}$ \\
PAD $(\mathrm{mmHg})^{\mathrm{b}}$ & $80(70-80)$ & $80(70-90)$ & $70(70-80)$ & $0,108^{*}$ \\
\hline
\end{tabular}

IMC: índice de masa corporal, CC: circunferencia de cintura, C-HDL: colesterol HDL, PAS: presión arterial sistólica, PAD: presión arterial diastólica.

a: promedio \pm desviación estándar; b: mediana (RI)

*: prueba de la mediana, **: prueba $\mathrm{t}$

Tabla 2. Prevalencia de síndrome metabólico y síndrome metabólico premórbido

\begin{tabular}{lcccccc}
\hline & & \multicolumn{2}{c}{ SM } & \multicolumn{2}{c}{ SMP } \\
Sexo & & Ausente & Presente & Ausente & Presente & Total \\
\hline Hombres & $\mathrm{n}(\%)$ & $34(46,6)$ & $14(56)$ & $36(47,4)$ & $12(54,5)$ & $48(49)$ \\
Mujeres & $\mathrm{n}(\%)$ & $39(53,4)$ & $11(44)$ & $40(52,6)$ & $10(45,5)$ & $50(51)$ \\
Total & $\mathrm{n}(\%)$ & $73(100)$ & $25(100)$ & $76(100)$ & $22(100)$ & $98(100)$ \\
\hline
\end{tabular}

SM (OMS 2009): Síndrome Metabólico, SMP (OMS 2010): Síndrome Metabólico Premórbido.

SM $\mathrm{pX}^{2}=0,416$, SMP: $\mathrm{pX}^{2}=0,553$

Al evaluar la distribución de los criterios del síndrome metabólico por sexo en los sujetos que lo padecían, se observó que la circunferencia de cintura $(p<0,001)$ y los triglicéridos $(p<0,001)$ fueron más frecuentes en mujeres, mientras que en los hombres fueron más frecuentes el colesterol HDL bajo $(p=0,001)$, la glucemia $(p=0,007)$ y la presión arterial elevada $(p=0,002)$. La misma tendencia se observó con el síndrome metabólico premórbido, a excepción de la glucemia elevada que fue más frecuente en las mujeres $(p=0,047)$ (Tabla 3).

Al relacionar el estado nutricional con el síndrome metabólico, en general se observó que los sujetos con bajo peso $(4,1 \% ; n=3)$ y normopeso $(27,4 \% ; n=20)$ y la mayoría de aquellos con sobrepeso $(45,2 \% ; n=33)$ no presentaron síndrome metabólico, a diferencia de los sujetos con obesidad, quienes en su mayoría sí presentaron (84\%; $\mathrm{n}=21)(\mathrm{p}<0,001)($ Tabla 4). 
Tabla 3. Distribución porcentual de los criterios del síndrome metabólico y síndrome metabólico premórbido por sexo entre los participantes con diagnóstico

\begin{tabular}{|c|c|c|c|c|c|c|}
\hline \multirow[b]{2}{*}{ Variables } & \multicolumn{3}{|c|}{ SM } & \multicolumn{3}{|c|}{ SMP } \\
\hline & $\begin{array}{l}\text { Hombres } \\
\%\left(\mathrm{IC}_{95 \%}\right)\end{array}$ & $\begin{array}{l}\text { Mujeres } \\
\%\left(\mathrm{IC}_{95 \%}\right)\end{array}$ & Valor $\mathrm{p}$ & $\begin{array}{l}\text { Hombres } \\
\%\left(\mathrm{IC}_{95 \%}\right)\end{array}$ & $\begin{array}{l}\text { Mujeres } \\
\%\left(\mathrm{IC}_{95 \%}\right)\end{array}$ & Valor p \\
\hline $\begin{array}{l}\text { Circunferencia } \\
\text { abdominal } \geq 102 \mathrm{~cm} \\
\text { (hombres) } 0 \geq 88 \\
\mathrm{~cm} \text { (mujeres) }\end{array}$ & 85,7 & 100 & $<0,001^{b}$ & 85,7 & 100 & $<0,001^{b}$ \\
\hline $\begin{array}{l}\mathrm{cHDL}<40 \mathrm{mg} / \mathrm{dl} \\
\text { (hombres) o }<50 \\
\mathrm{mg} / \mathrm{dl} \text { (mujeres) }\end{array}$ & 64,3 & 54,5 & $0,001^{a}$ & 64,3 & 54,5 & $0,001^{a}$ \\
\hline $\begin{array}{l}\text { Glucemia en } \\
\text { ayunas } \geq 100 \mathrm{mg} / \mathrm{dl} \\
\text { o tto farmacológico }\end{array}$ & 28,6 & 27,3 & $0,007^{b}$ & 16,7 & 20 & $0,047^{b}$ \\
\hline $\begin{array}{l}\text { Triglicéridos } \geq 150 \\
\mathrm{mg} / \mathrm{dl}\end{array}$ & 85,7 & 100 & $<0,001^{b}$ & 85,7 & 100 & $<0,001^{b}$ \\
\hline $\begin{array}{l}\mathrm{PAS} \geq 130 \circ \\
\mathrm{PAD} \geq 85 \mathrm{mmHg} \circ \\
\text { tto farmacológico }\end{array}$ & 71,4 & 54,5 & $0,002^{c}$ & 71,4 & 54,5 & $0,002^{c}$ \\
\hline
\end{tabular}

Tabla 4. Relación entre el estado nutricional y el síndrome metabólico en la muestra

\begin{tabular}{lcccc}
\hline & \multicolumn{5}{c}{ SM } \\
\hline Estado nutricional & & Ausente & Presente & Total \\
Bajo peso & $\mathrm{n}(\%)$ & $3(4,1)$ & $0(0)$ & $3(3,1)$ \\
Normopeso & $\mathrm{n}(\%)$ & $20(27,4)$ & $0(0)$ & $20(20,4)$ \\
Sobrepeso & $\mathrm{n}(\%)$ & $33(45,2)$ & $4(16)$ & $37(37,8)$ \\
Obesidad & $\mathrm{n}(\%)$ & $17(23,3)$ & $21(84)$ & $38(38,8)$ \\
Total & $\mathrm{n}(\%)$ & $73(100)$ & $25(100)$ & $98(100)$ \\
\hline
\end{tabular}

SM (OMS 2009): Síndrome Metabólico

$\mathrm{pX}^{2}<0,001$

Al discriminar por sexo, se observó que la mayoría de las mujeres y de los hombres presentaron obesidad hallándose un mayor porcentaje de síndrome metabólico $(p=0,001)$ (Tabla 5). 
Tabla 5. Relación entre el estado nutricional y el síndrome metabólico según sexo

\begin{tabular}{|c|c|c|c|c|c|c|c|}
\hline \multirow{3}{*}{$\begin{array}{l}\text { Estado } \\
\text { nutricional }\end{array}$} & & \multirow{2}{*}{\multicolumn{2}{|c|}{$\frac{\text { Hombres }}{\text { SM }}$}} & \multicolumn{4}{|c|}{ Mujeres } \\
\hline & & & & & & & \\
\hline & & Ausente & Presente & Total & Ausente & Presente & Total \\
\hline Bajo peso & $\mathrm{n}(\%)$ & $0(0)$ & $0(0)$ & $0(0)$ & $3(7,7)$ & $0(0)$ & $3(6)$ \\
\hline Normopeso & $\mathrm{n}(\%)$ & $5(14,7)$ & $0(0)$ & $5(10,4)$ & $15(38,5)$ & $0(0)$ & $15(30)$ \\
\hline Sobrepeso & $\mathrm{n}(\%)$ & $20(58,8)$ & $2(14,3)$ & 22 & $13(33,3)$ & $2(18,2)$ & $15(30)$ \\
\hline Obesidad & $\mathrm{n}(\%)$ & $9(26,5)$ & $12(85,7)$ & 21 & $8(20,5)$ & $9(81,8)$ & $17(34)$ \\
\hline Total & $\mathrm{n}(\%)$ & $34(100)$ & $14(100)$ & 48 & 39 (100) & $11(100)$ & $50(100)$ \\
\hline
\end{tabular}

SM (OMS 2009): Síndrome Metabólico

Si bien evaluar la prevalencia del síndrome metabólico según los criterios de la IDF no formaba parte de los objetivos del estudio, fue realizado teniendo en cuenta que utiliza los puntos de corte de circunferencia de cintura adaptados a la población de América Latina.

La prevalencia fue del $32,7 \%$, al discriminar por sexo se observó que el $59,4 \%(n=19)$ de los hombres y el 40,6\% $(n=13)$ de las mujeres presentaban síndrome metabólico.

Al definir la circunferencia de cintura según criterio de la IDF, la mayoría presentó valores aumentados $(76,5 \%)$, en cambio al utilizar el criterio del síndrome metabólico premórbido (OMS 2010) la mayoría presentó valores en rango adecuado $(54,1 \%)$.

\section{SCUSIÓN}

En los trabajos de investigación que evalúan el síndrome metabólico son más utilizados los criterios de la Federación Internacional de Diabetes (IDF), sin embargo en los últimos años la OMS ha realizado consensos concluyendo en el 2010 en la actual definición del síndrome metabólico, la cual excluye a aquellos sujetos con diagnóstico de diabetes mellitus y con antecedente de enfermedad coronaria, considerándolo como una condición premórbida a fin de poder llevar a cabo acciones de prevención primaria.

En el presente estudio se utilizó la definición del síndrome metabólico premórbido de la OMS 2010 para evaluar la prevalencia del síndrome metabólico en 98 sujetos de los cuales 48 fueron hombres y 50 mujeres con una mediana de edad de 46 y 44 años respectivamente.

Al analizar las características antropométricas y clínicas, se encontraron valores más altos de peso y talla $(p<0,001)$, circunferencia de cintura $(p<0,001)$, triglicéridos $(p=0,043)$ y presión arterial sistólica $(p=0,005)$ y disminuido el colesterol HDL $(p<0,001)$ en hombres.

Un trabajo publicado por Oliveira $C$ et al. que evalúa la relación entre el fenotipo circunferencia de cintura-triglicéridos elevados con cambios metabólicos en adultos, mostró que aquellos hombres que presentaron circunferencia de cintura-triglicéridos aumentados, presentaron significativamente disminuido el c-HDL, y elevados otros factores de riesgo cardiovascular como son el colesterol y tejido adiposo visceral, misma tendencia se observó en las mujeres sumándose además la presión arterial sistólica elevada (14). 
Al evaluar el estado nutricional a través del índice de masa corporal por sexo, se observó un mayor porcentaje de sobrepeso y obesidad en hombres que en mujeres.

Un estudio publicado por Gierach et al. revela la existencia de una fuerte correlación lineal entre la circunferencia de cintura y el IMC $(p<0,01)$, lo que podría explicar los valores elevados tanto de circunferencia de cintura como del índice de masa corporal en hombres. Si bien sabemos que la circunferencia de cintura se relaciona con el síndrome metabólico, por ende el estado nutricional también podría estar relacionado (15).

Al analizar la distribución de los criterios del síndrome metabólico (OMS 2.009) por sexo en los sujetos que lo padecían, fueron más frecuentes los criterios de circunferencia de cintura $(p<0,001)$ y triglicéridos $(p<0,001)$ en las mujeres, mientras que en los hombres fueron más frecuentes el colesterol HDL bajo $(p=0,001)$, la glucemia $(p=0,007)$ y la presión arterial elevadas $(p=0,002)$. Misma tendencia se observó con el síndrome metabólico premórbido (OMS 2010), a excepción de la glucemia que fue mayor en las mujeres $(p=0,047)$, esto podría ser debido a la limitación del criterio de la glucemia en la definición del síndrome metabólico premórbido (>100 $\leq 126 \mathrm{mg} / \mathrm{dl}$ ), reduciendo el número de hombres con criterio de glucemia elevada.

La diferencia de criterios del síndrome metabólico entre hombres y mujeres puede estar relacionada al estilo de vida. Un estudio realizado por Cassaza $\mathrm{K}$ et al. en una población infantil concluyó que la dieta al igual que la actividad física estaban muy relacionadas con los componentes del síndrome metabólico, influyendo la alta ingesta de hidratos de carbono negativamente sobre la circunferencia de cintura, los niveles de triglicéridos y glucemia, y la actividad física moderada a intensa de manera positiva mejorando los niveles de c-HDL (16).

Al determinar la prevalencia del síndrome metabólico fue del 25,5\% $(n=25)$, el síndrome metabólico premórbido la redujo a un 22,4\% $(n=22)$. Misma tendencia se observó en dos estudios diferentes realizados por Bergés et al., donde se presenta una reducción de la prevalencia utilizando el síndrome metabólico premórbido, delimitando a la población para la prevención primaria $(8,17)$.

La prevalencia fue mayor en los hombres que en las mujeres al igual que en otros estudios realizados en España y Estados Unidos $(17,18)$.

Al relacionar el estado nutricional con el síndrome metabólico, en general se observó que los sujetos con bajo peso y normopeso, y la mayoría de aquellos con sobrepeso no presentaron síndrome metabólico, estando presente en la mayoría de los sujetos con obesidad $(p<0,001)$.

La obesidad se asocia a una serie de desajustes metabólicos como los observados en un estudio realizado en adolescentes por Saldaña $C$ et al., donde tanto la circunferencia de cintura, la prensión arterial sistólica y diastólica, y los triglicéridos fueron significativamente mayores y el c-HDL significativamente menor en los sujetos con obesidad, todos ellos componentes del síndrome metabólico lo que supone una mayor prevalencia en dicho grupo $(19,20)$.

Si bien el mayor porcentaje de sujetos con síndrome metabólico eran obesos, también hubo, aunque en menor número, personas obesas sin diagnóstico de síndrome metabólico.

Es sabido que la obesidad se caracteriza por la inflamación del tejido adiposo, lo que predispone a la diabetes mellitus, hipertensión arterial y enfermedades cardiovasculares. Por otro lado, un estudio español determinó la existencia de un subconjunto de individuos obesos que parece estar protegido contra la resistencia a la insulina, mostrando por ende menos inflamación y menos trastornos predisponentes. Este subconjunto es conocido como ISO (obesos insulino sensibles) entre sus características resaltan: menor insulina en ayunas, menores niveles de HbA1c, tolerancia normal a la glucosa, no son hipertensos, menores triglicéridos circulantes, aumento del c-HDL, lo que sugiere una mayor capacidad para almacenar lípidos en su tejido adiposo 
subcutáneo y menos en la grasa visceral, hígado y músculo, órganos en los que la acumulación de lípidos intracelulares se asocian a resistencia a la insulina. Se necesitan más investigaciones para descubrir el mecanismo exacto que protege a este subconjunto de obesos contra la inflamación del tejido adiposo (21).

Al analizar la relación entre el estado nutricional y el síndrome metabólico por sexo se observó que la mayoría de las mujeres con sobrepeso y de los hombres con obesidad presentaron un mayor porcentaje de síndrome metabólico $(p=0,001)$.

Se analizó presencia del síndrome metabólico en mujeres con edad inferior a 50 años siendo la mayoría $(n=31)$ y en aquellas con edades mayores o igual a 50 años encontrándose en estas últimas significativamente elevada $(p<0,001)$.

El mayor porcentaje de síndrome metabólico en mujeres con sobrepeso podría deberse a que en promedio a partir de los 50 años tiene lugar en la mujer la menopausia, produciendo cambios desfavorables en el perfil cardiovascular alterando el perfil lipídico, aumentando la fracción del colesterol ligado a la lipoproteína de baja densidad (c-LDL) y los triglicéridos y disminuyendo el colesterol ligado a la lipoproteína de alta densidad (c$\mathrm{HDL}$ ), elevando la presión arterial, principalmente la diastólica. Estos cambios se deben a la disminución del estrógeno, hormona responsable del aumento del c-HDL y de la relajación vascular. Estudios han mostrado una relación entre la menopausia y el grado de adiposidad, la reducción de los niveles de estrógeno junto a un relativo hiperandrogenismo son responsables del aumento de peso y cambios en la distribución del tejido adiposo. La tasa metabólica en reposo junto con la actividad física se reduce en las mujeres postmenopáusicas, deduciendo así un mayor porcentaje de sobrepeso en mujeres en edad menopaúsica, y por ende un mayor porcentaje de síndrome metabólico (22).

Se analizó también la prevalencia del síndrome metabólico según los criterios de la IDF a fin de comparar con el síndrome metabólico premórbido. La prevalencia fue mayor con un $32,7 \%$, esto se debe a las diferencias que existen en los puntos de corte para la determinación de la circunferencia de cintura, siendo elevados los utilizados por la OMS cuando se implementan en América Latina, minimizando la obesidad abdominal, lo que descarta personas en riesgo de padecer enfermedad cardiovascular y diabetes mellitus (23).

Al definir la circunferencia de cintura según criterio de la OMS la mayoría presentó adecuada $(54,1 \%)$, en cambio, al utilizar el criterio de la IDF la mayoría presentó aumentada $(76,5 \%)$, siendo mayor el riesgo para desarrollar enfermedades cardiovasculares.

Sería interesante estudiar la prevalencia del síndrome metabólico en una muestra más representativa a fin de determinar con precisión la situación salud/enfermedad de nuestra población.

Como fortaleza del trabajo cabe destacar la falta de publicaciones que revelen la prevalencia del síndrome metabólico en adultos a nivel nacional en Paraguay, siendo de gran importancia para identificar los riesgos para el desarrollo a futuro de diabetes mellitus o enfermedad cardiovascular.

Los resultados del estudio, si bien representan los hallazgos de un tamaño muestral mínimo para lograr los fines de la investigación, demostró que el estado nutricional y el síndrome metabólico se incrementan de manera proporcional, lo que permite concluir que existe relación entre el estado nutricional y el síndrome metabólico. 


\section{REFERENCIAS BI BLIOGRÁFICAS}

1. Organización Mundial de la Salud. Informe sobre la situación mundial de las enfermedades no transmisibles 2010: Resumen de orientación. Ginebra: OMS; 2011.

2. Centro de Noticias ONU: La población mundial alcanza hoy los 7.000 millones / Internet/. Naciones Unidas.Son.,/acceso 3 Enero2014]. Disponible en: http:// www.un.org/spanish/News/story.asp? newsID $=22135 \#$. UvB2AfI5M40

3. World Health Organization. Burden: mortality, morbidity and risk factors / Internet/. En: World Health Organization. Global status report on noncommunicable diseases 2010. Geneva: WHO; 2011. p. 931./citado 3 Enero 2014/. Disponible en: h t t p : / / a p s.w h o. in t/iris / bitstream/10665/44579/1/9789240686458_ eng.pdf

4. Reaven GM. Bathing lecture 1988: Role of insulin resistance in human disease. Diabetes.1988; 37(12):1595-607.

5. Rosas Guzmánn J, González Chávez A, Aschner P, Bastarrachea R, editores. Consenso latinoamericano de la Asociación Latinoamericana de Diabetes (ALAD): Epidemiología, diagnóstico, control, prevención y tratamiento del síndrome metabólico en adultos. Rev ALAD. 2010; 18 (1):25-44.

6. Liu Z. Obesity and metabolic syndrome. N A J Med Sci. 2009; 2(3):88-9.

7. Medina Palomino F. Apuntes sobre los factores de riesgo de enfermedad cardiovascular /Editorial/. Rev Med Hered. 2012; 23(3):145-7.

8. Fernández-Bergés $D$, Redondo FJF, Lozano L, Pérez-Castán JF, Sanz H, Cabrera De León $A$, et al. Prevalencia de síndrome metabólico según las nuevas recomendaciones de la OMS: Estudio HERMEX. Gac Sanit. 2011; 25(6):519-24.

9. Zimmet $P$, Alberti G, Serrano $M$. Nueva definición mundial del síndrome metabólico propuesta por la Federación Internacional de Diabetes: fundamento y resultados. Rev Esp Cardiol. 2005; 58(12):1371-6.

10.Uchospital.edu. Síndrome metabólico. The University of Chicago Medicine (sede Web). Chicago: UCM [acceso 4 Feb 2014]. Disponible en: http://www.uchospitals.edu/ online-library/content $=$ S08351
11.Garrido C. Sociedad: Obesos, pero muy sanos /comentarios/. /Internet/. Madrid. / acceso 5 Dic 2013]. Disponible en: http:// www.abc.es/20101011/sociedad/obesossanos-201010111205.html

12. Cañete F. Primera encuesta nacional de factores de riesgo y enfermedades no transmisibles Paraguay 2011. Asunción: Ministerio de Salud Pública y Bienestar Social, Dirección de Vigilancia de Enfermedades No Trasmisibles; 2011.

13. Riveros Sasaki K, Alderete Peralta V, Sánchez Bernal S. Frecuencia de elementos del síndrome metabólico en adolescentes de un colegio público. Pediatr. (Asunción). 2012; 39(1):13-9.

14.Cunha de Oliveira C, Carneiro Roriz AK, Eickemberg M, Barreto Medeiros JM, Ramos L. Hypertriglyceridemic waist phenotype: Association with metabolic disorders and visceral fat in adults. Nut Hosp. 2014;30 (1):25-31.

15. Gierach M, Gierach J, Ewertowska M, Arndt A, Junik R. Correlation between body mass index and waist circumference in patients with metabolic syndrome. ISRN Endocrinology. 2014; 2014:1-6.

16.Casazza K, Dulin-Keita A, Gower BA, Fernandez JR. Differential influence of diet and physical activity on components of metabolic syndrome in a multiethnic simple of children. J Am Diet Assoc. 2009; 109(2): 236-44.

17.Fernández-Bergés $D$, Cabrera de León $A$, Sanz H, Elosua R, Guembe MJ, Alzamora M, et al. Síndrome metabólico en España: Prevalencia y riesgo coronario asociado a la definición armonizada y a la propuesta por la OMS: Estudio DARIOS. Rev Esp Cardiol. 2012; 65(3):241-8.

18. Ford ES, Li C, Zhao G. Prevalence and correlates of metabolic syndrome based on a harmonious definition among adults in the US. J Diabetes. 2010; 2(3):180-93.

19. Contreras-Leal EA, Santiago-García J. Obesidad, síndrome metabólico y su impacto en las enfermedades cardiovasculares. Rev Biomed. 2011; 22:103-15.

20.Cardozo-Saldaña GC, Yamamoto-Kimura L, Medina-Urrutia A, Posadas-Sánchez R, Caracas-Portilla NA, Posadas-Romero C. Exceso de peso y síndrome metabólico en adolescentes de la ciudad de México. Arch. Cardiol. Méx. 2010; 80(1):12-8. 
21. Gauthier MS, Ruderman NB. Adipose tissue inflammation and insulin resistance: All obese humans are not created equal. Biochem J. 2010; 430(2):e1-4.

22.Soca PEM, Rivas Estévez M, Sarmiento Teruel Y, Mariño Soler AL, Marrero Hidalgo M. Características clínicas, antropométricas y de laboratorio en mujeres con menopausia /Internet/. En: Primer congreso virtual de ciencias morfológicas; Primera jornada científica de la cátedra Santiago Ramón y Cajal. La Habana: MorfoVirtual; 2012. /citado 3 Feb 2014/. Disponible en: http://www.morfovirtual2012.sld.cu/ index.php/morfovirtual/2012/paper/ view/131/210
23. Jolliffe C, Janssen I. Development of agespecific adolescent metabolic syndrome criteria that are linked to the Adult Treatment Panel III and International Diabetes Federation criteria. J Am Coll Cardiol. 2007; 49 (8):891-8. 